\title{
Review
}

\section{Advances in studies on human papillomavirus infection and oropharyngeal cancer}

Zhitao Guo

Department of Emergency Medicine, Tianjin Xiqing Hospital, Tianjin China

\author{
Keywords \\ Oropharyngeal neoplasms; Human \\ papillomavirus; Prognosis; Vaccine; Review \\ literature
}

Correspondence

Zhitao Guo,

E-mail: guozhitaotj@sina.com

DOI: 10.1515/ii-2017-0113

\begin{abstract}
Objective: This study summarizes research progress on human papillomavirus (HPV) infection and carcinogenesis mechanism, curative effect, and prognosis of oropharyngeal epithelium.

Methods: By using Medline and PubMed database retrieval system and "HPV, oropharyngeal cancer" as keywords, we searched relevant literature from January 1998 to June 2012 and incorporated results into the following criteria: 1) biological characteristics of HPV; 2) carcinogenic mechanism and route of HPV transmission; 3) HPV infection rate in oropharyngeal cancer; 4) HPV infection and prognosis of oropharyngeal cancer; and 5) HPV vaccine. A total of 38 articles were analyzed according to incorporated criteria. Results: HPV mainly infects oral mucosa through direct mouth-genital contact. Among general populations, oropharyngeal cancer tissues present higher HPV infection rate than oral mucosal epithelium. Polymerase chain reaction-based detection shows the highest sensitivity and is most widely used in HPV detection. High-risk HPV16/18 is a commonly detected type. HPV-positive oropharyngeal cancer is an independent subtype, displaying unique molecular biological and clinical features. Tumor tissues rare exhibit P53 mutation. Oropharyngeal cancer patients are sensitive to radiotherapy and chemotherapy and display long-term prognosis. Preventive effects of HPV vaccine on oropharyngeal cancer still require elucidation.

Conclusion: HPV infection is an important risk and independent prognostic factor of oropharyngeal cancer.
\end{abstract}

Oropharyngeal cancer refers to tumors that occur in tonsils, sublingual part and soft palate of the tongue, and other parts. Squamous cell carcinoma is the main pathological type and accounts for over $80 \%$ of all pathological types. Alcohol and smoking represent the main risk factors of oropharyngeal cancer, but $15 \%$ to $20 \%$ of patients with oropharyngeal cancer do not feature a smoking history. This finding suggests that other risk factors may be involved in development of oropharyngeal tumors ${ }^{[1]}$. In recent years, the number of smokers in Europe and the United States gradually reduced, and overall incidence rate of head and neck neoplasms also showed a downward trend each year; incidence rate of oropharyngeal cancer continually increased among young men ${ }^{[2,3]}$. These results also prove that other risk factors may be involved in development of oropharyngeal tumors. In 1983, Syrjanen et al. ${ }^{[4]}$ first reported that human oral papillomavirus (HPV) infection was detected in oral squamous cell carcinoma and later discovered that HPV infection also exist in oropharynx, hypopharynx, throat, and other tumors; however, oropharyngeal cancer yields significantly higher positive rate than tumors in the head and neck and other parts ${ }^{[5,6]}$. Current research confirmed that HPV-positive oropharyngeal cancer is an independent subtype, exhibiting unique molecular biological and clinical features ${ }^{[2]}$. This study reviews research progress on HPV infection and carcinogenesis mechanism, prognostic relevance, and vaccine prevention of oropharyngeal cancer.

\section{HPV structure and classification}

HPV belongs to the nipple polygonal vacuolar virus family of papillomavirus genus. The virus features a closed circular 
double-stranded DNA, and its virus particles consist of nucleic acids and capsid proteins. HPV genome can be divided into three regions based on function: 1) early stage region, which is responsible for coding viral replication and relevant proteins for host-cell transformation. Proteins encoded by E 6 and $\mathrm{E} 7$ genes are related to malignant transformation of host cells and are major carcinogens of HPV; 2) late stage region, which is responsible for coding virus capsid protein; and 3) long-term control region, which is mainly responsible for regulating viral DNA replication and transcription ${ }^{[7,8]}$.

HPV features high variety of species and tissue specificity. This virus mainly infects the human skin and mucous membrane tissues, causing benign lesions or malignant tumors. Based on type and risk level of cervical cancer, HPV can be divided into high-risk, probable high-risk, and low-risk ${ }^{[9]} \mathrm{HPV}$ : among these groups, low-risk types include HPV6, $11,40,42,43,44,54,61,70,72,81$, and CP6108, which often cause genital warts and respiratory and gastrointestinal papilloma; probable high-risk types include HPV26, 53, and 66; high-risk types comprise HPV16, 18, 31, 33, 35, 39, 45, $51,52,56,58,59,68,73$, and 82 , which are associated with cervical intraepithelial neoplasia, cervical cancer, and other anus genital tumors ${ }^{[8,10]}$.

\section{HPV infection rate in oral mucosa and oropharyngeal cancer tissues among healthy people}

HPV infection may also affect oral mucosa of healthy people. A systematic review in 2010 showed that among healthy individuals, HPV infection rate reached 4.5\% (182/4 070) for all types and 3.5\% (155/4 441) for high-risk types, of which HPV16 was the most common with an infection rate of $1.3 \%(51 / 3977)^{[11]}$. In 2012, a large sample of HPV infection among healthy people in the United States showed that oral HPV infection rate totaled $6.9 \%$ among healthy people aged 14 to 69 (408/5579); infection rate of HPV16 reached $1.0 \%(48 / 5579)$. HPV infection rate in males was higher than in females, and risk of oral HPV infection is positively correlated with the number of sexual partners and daily smoking ${ }^{[12]}$.

Oropharyngeal carcinoid epithelial tissues manifest significantly higher HPV infection rate than normal epithelial tissues. A meta-analysis in 2010 showed $41 \%$ HPV-positive rate in oropharyngeal cancer ${ }^{[13]}$. HPV16 is a primary infection type in oropharyngeal cancer and accounts for over $90 \%$ of all HPV infection, followed by HPV18, which accounts for $5 \%$ of such cases ${ }^{[14,15]}$. Different literature reported significantly different HPV detection rates of oropharyngeal cancer. Positive rate ranges from $8 \%$ to $100 \%$. This result is related to geographical origin, location of dissection, sensitivity of detection method to samples, and type and number of samples ${ }^{[16]}$. Detection methods based on nucleic acid amplification [e.g., polymerase chain reaction (PCR), fluorescent quantitative PCR, and PCR joint in situ hybridization,) are highly sensitive and widely used ${ }^{[17,18]}$.

HPV is mainly transmitted through sexual contact, but it can also be transmitted through daily contact. Infection site is mainly through anus genitalia. Mechanism of oral mucosa infection with HPV remains to be clarified. Three possible ways were formulated: 1) birth canal transmission: evidence comes from children's recurrent respiratory papilloma, which occurs at the larynx. Newborns are infected with HPV from mother's birth canal during delivery. However, whether oral mucosal HPV infection can continue from neonatal period to adult still requires confirmation. 2) Self transmission: women that are HPV positive in the birth canal display high risks of oral mucosal HPV infection, and risk of oropharyngeal neoplasms in men with genital warts increases by two times, presumably through hands, which transmit HPV to oral cavity ${ }^{[19]}$. 3) Oral sex transmission: direct contact between mouth and genitals is the main route of transmission for oral HPV infection. Risk of oral HPV infection increases with the number of oral sex partners ${ }^{[20]}$. Oropharyngeal cancer patients with oral sex experience show significantly higher HPV detection rate in tumor tissues than patients without oral sex experience. Among the youth population in Europe, incidence rate of oral cancer associated with HPV infection increases gradually, and it is presumably related to increase in oral sex among considered individuals ${ }^{[21]}$. HPV possibly orally spreads among individuals through kissing ${ }^{[12]}$.

\section{Carcinogenic mechanism of HPV- induced oropharyngeal epithelial cancer}

HPV is exposed to basal cells through microlesions on the skin or mucous membranes. After the virus removes its capsid protein, its DNA enters host nucleus in bound or unbound form. In most people, HPV infection is transient and latent and can be cleared by host autoimmune system 
after one to two years ${ }^{[22]}$. In benign lesions and precancerous lesions, high-risk HPV exists in unbound form. In malignant lesions, virus genes bind to host cell chromosomes. The protein encoded by HPV E6 gene degrades wild-type p53 protein through ubiquitination, whereas the protein encoded by E7 gene binds with cell cycle negative regulation protein, the retinoblastoma $\mathrm{Rb}$ protein, and inactivates its function. Inactivation of two important tumor suppressor proteins, p53 protein and $\mathrm{Rb} 2$, leads to deregulation of cell cycle, resulting in accelerated cell proliferation and division and promoted cell malignant transformation ${ }^{[8]}$.

HPV-positive oropharyngeal tumor cells possess low viral content of only dozens of copies/cell, but viral genome is generally integrated into host chromosome ${ }^{[23]}$. During the 10 to 20 year-course of disease from normal epithelium infected with high-risk HPV to eventually development of malignant tumor, integration of virus occurs during the early stage. Host cells integrate HPV E6 and E7 genes, display selective growth advantage, and may possibly become cancerous during long-term chronic infection.

\section{HPV infection and oropharyngeal tumor prognosis and its mechanism}

Most patients with HPV-positive oropharyngeal cancer do not present histories of smoking and drinking, and tumors exhibit unique molecular biological, epidemiological, and clinical characteristics and good prognosis and constitute a subgroup of oropharyngeal tumors ${ }^{[2,24]}$. HPV infection is an independent prognostic factor for overall survival and disease-free survival of oropharyngeal cancer. Regardless of whether patients underwent surgical resection or chemotherapy radiotherapy, HPV-positive tumors in tumor tissues constantly suggest high survival rates ${ }^{[25]}$. Compared with HPV-negative oropharyngeal cancer, although HPVpositive oropharyngeal cancer shows onset at an early age, poor histopathological differentiation, and early local metastasis, it is more sensitive to radiotherapy and chemotherapy and presents low local recurrence rate, thus indicating good survival prognosis ${ }^{[23,24]}$. Higher number of HPV copies in tumor tissues indicates longer overall survival and disease-free survival of patients and significantly reduced probability of tumor recurrence ${ }^{[26]}$. Although HPV-positive patients with oropharyngeal cancer show good prognosis, when patients claim history or previous history of continuous smoking, their survival, death risk, recurrence interval, and other indicators are inferior to those of nonsmoking $\mathrm{HPV}$-positive patients with oropharyngeal cancer ${ }^{[2,27,28]}$. In assessment of oropharyngeal cancer prognosis, in addition to detecting HPV gene fragments in tumor tissues, we can also detect expression of p16 protein in tumors by immunohistochemical method. p16 protein is negatively regulated by upstream $\mathrm{Rb}$, whereas HPV E7 oncoproteins can inactivate $\mathrm{Rb}$. This condition results in increased $\mathrm{p} 16$ expression. Thus, p16 overexpression in oropharyngeal tumor tissues serves as molecular marker of HPV infection and indicator of good patient prognosis ${ }^{[29]}$. HPV-positive patients with integrated 16 protein overexpression display significantly better survival prognosis than HPV-negative individuals with low expression of p16 protein ${ }^{[30]}$.

Further studies should clarify exact sensibility mechanism of patients with HPV-positive oropharyngeal cancer to radiotherapy and chemotherapy, which both can revive p53 protein function and enhance local immune responses in tumors ${ }^{[14]}$. Mutation or inactivation of p53 gene is an important factor during tumor formation. In oropharyngeal tumor tissues of $\mathrm{HPV}$-positive patients, p53 protein content is low and mostly corresponds to the wild type. p53 mutation is very common in oropharyngeal tumor tissues of HPVnegative patients. During in vitro intervention experiment on cervical cancer SiHa cells of HPV16-positive patients, cisplatin inhibited expression of E6E7 protein in HPV16, increased p53 protein expression, and induced p53dependent apoptosis pathway, suggesting that wild-type state of p53 in HPV-positive tumors influences sensitivity to radiotherapy and chemotherapy [31]. Radiotherapy and chemotherapy can increase immunogenicity of HPV-positive tumor cells, induce local immune responses, and kill tumor cells while removing viruses ${ }^{[32,33]}$.

\section{HPV vaccine with oropharyngeal cancer}

Currently, two kinds of HPV-preventive vaccines are listed in the United States: One is tetravalent vaccine Gardasil ${ }^{\odot}$ , which was developed by Merck mainly for HPV6, 11, 16, and 18 . The other is bivalent vaccine Cervarix ${ }^{\circledR}$ developed by GlaxoSmithKline and primarily targets HPV16 and 18 . Considering that healthy men and women may be both infected by HPV, in February 2012, the American Academy of Pediatrics released a new HPV Vaccine Guideline recommending that boys and girls should be vaccinated when 
they reach 11 or 12 years old; boys can only be vaccinated with tetravalent HPV vaccine, and girls can be vaccinated with both bivalent or tetravalent HPV vaccines ${ }^{[34]}$. These vaccines provide protection against common HPV16 and HPV18 infection in oropharyngeal cancer. However, further studies should confirm their preventive effects on high-risk oropharyngeal cancer population.

\section{Problems and Prospects}

HPV infection is an important pathogenic factor of oropharyngeal cancer. Oral-genital direct contact is the main route of HPV infection in oral mucosa. Patients with HPV-positive oropharyngeal cancer are sensitive to radiotherapy and chemotherapy and exhibit good long-term prognosis. Relevant research on HPV and oropharyngeal cancer cannot explain causes of higher HPV infection rate in men than women. Additional research should determine sensibility mechanism of patients with HPV-positive oropharyngeal cancer to radiotherapy and chemotherapy, whether they should be given active treatment, and whether extensive vaccination of $\mathrm{HPV}$ vaccine can ultimately reduce incidence of HPV infection-related oropharyngeal cancer. Answers to these issues will provide important implications for prevention and screening of high-risk groups with oropharyngeal cancer. Findings can also aid in treatment selection for patients and prognosis evaluation.

\section{Declarations}

\section{Acknowledgements}

No.

\section{Competing interests}

The author declares that he has no competing interest.

\section{Authors' contributions}

ZT Guo made the literature analysis and wrote, discussed and revised the manuscript of this review.

\section{References}

1 Gillison ML, Koch WM, Capone RB, et al. Evidence for a causal association between human papillomavirus and a subset of head and neck cancers. J Natl Cancer Inst,2000,92(9):709-720.

2 Marur S, D'Souza G, Westra WH, et al. HPV-associated head and neck cancer:a virus-related cancer epidemic. Lancet Oncol, 2010,11(8):781-789.

3 Sturgis EM, Cinciripini PM. Trends in head and neck cancer incidence in relation to smoking prevalence:an emerging epidemic of human papillomavirus-associated cancers?. Cancer, 2007,110(7):1429-1435.

4 Syrjanen K, Syrjanen S, Lamberg M, et al. Morphological and immunohistochemical evidence suggesting human papillomavirus (HPV) involvement in oral squamous cell carcinogenesis. Int J Oral Surg, 1983,12(6):418-424.

5 D'Souza G, Kreimer AR, Viscidi R, et al. Case-control study of human papillomavirus and oropharyngeal cancer. N Engl J Med, 2007,356(19):1944-1956.

6 Chaturvedi AK, Engels EA, Pfeiffer RM, et al. Human papillomavirus and rising oropharyngeal cancer incidence in the United States. J Clin Oncol,2011,29(32):4294-4301.

7 Psyrri A, Dimaio D. Human papillomavirus in cervical and headand-neck cancer. Nat Clin Pract Oncol, 2008,5(1):24-31.

8 Zur HH. Papillomaviruses and cancer: from basic studies to clinical application. Nat Rev Cancer, 2002,2(5):342-350.

9 Munoz N, Bosch FX, de Sanjose S, et al. Epidemiologic classification of human papillomavirus types associated with cervical cancer. N Engl J Med, 2003,348(6):518-527.

10 Ni X, Lei L, Chen Q et al. Discussion of diagnostic value of high-risk human papillomavirus in cervical cancer and precancerous lesions . Chinese Journal of Cancer Prevention, 2010, 17 (22): 1886- 1887.

11 Kreimer AR, Bhatia RK, Messeguer AL, et al. Oral human papillomavirus in healthy individuals: a systematic review of the literature. Sex Transm Dis, 2010,37(6):386-391.

12 Gillison ML, Broutian T, Pickard RK, et al. Prevalence of oral HPV infection in the United States, 2009-2010. JAMA, 2012,307(7):693-703.

13 Dayyani F, Etzel CJ, Liu M, et al. Meta-analysis of the impact of human papillomavirus (HPV) on cancer risk and overall survival in head and neck squamous cell carcinomas (HNSCC). Head Neck Oncol, 2010,2:15.

14 Lowy DR, Munger K. Prognostic implications of HPV in oropharyngeal cancer. N Engl J Med, 2010,363(1):82-84.

15 Kreimer AR, Villa A, Nyitray AG, et al. The epidemiology of oral HPV infection among a multinational sample of healthy men. Cancer Epidemiol Biomarkers Prev, 2011,20(1):172-182.

16 Termine N, Panzarella V, Falaschini S, et al. HPV in oral squamous cell carcinoma vs head and neck squamous cell carcinoma biopsies: a metaanalysis (1988-2007). Ann Oncol, 2008,19(10):1681-1690.

17 Li X, Gao L, Li H, et al. Human papillomavirus infection and laryngeal cancer risk: a systematic review and meta-analysis. J Infect Dis, 2013,207(3):479-488.

18 Venuti A, Paolini F. HPV detection methods in head and neck cancer. Head Neck Pathol, 2012,6 (Suppl 1):S63-S74.

19 Campisi G, Giovannelli L. Controversies surrounding human papilloma 
virus infection, head \& neck vs oral cancer,implications for prophylaxis and treatment. Head Neck Oncol, 2009,1:8.

20 D'Souza G, Agrawal Y, Halpern J, et al. Oral sexual behaviors associated with prevalent oral human papillomavirus infection. J Infect Dis, 2009,199(9):1263-1269.

21 Jemal A, Bray F, Center MM, et al. Global cancer statistics.CA Cancer J Clin, 2011,61(2):69-90.

22 Schiffman M, Castle PE, Jeronimo J, et al. Human papillomavirus and cervical cancer. Lancet, 2007,370(9590):890-907. mp HC, Manni JJ, Haesevoets A, et al. Marked differences in survival rate between smokers and nonsmokers with HPV 16-associated tonsillar carcinomas. Int J Cancer, 2008,122(12):2656-2664.

24 Byrd JK, Wilhoit CS, Fordham MT, et al. Predicting HPV status in head and neck cancer: The predictive value of sociodemographic and disease characteristics. Arch Otolaryngol Head Neck Surg, 2012, 138(12):1155-1159.

25 Ang KK, Harris J, Wheeler R, et al. Human papillomavirus and survival of patients with oropharyngeal cancer. N Engl J Med, 2010,363(1):24-35. Cohen MA, Basha SR, Reichenbach DK, et al. Increased viral load correlates with improved survival in HPV-16-associated tonsil carcinoma patients. Acta Otolaryngol, 2008,128(5):583-589.

27 Maxwell JH, Kumar B, Feng FY, et al. Tobacco use in human papillomavirus-positive advanced oropharynx cancer patients related to increased risk of distant metastases and tumor recurrence. Clin Cancer

\section{Res, 2010,16(4):1226-1235.}

28 Gillison ML, Zhang Q Jordan R, et al. Tobacco smoking and increased risk of death and progression for patients with p16-positive and p16negative oropharyngeal cancer. J Clin Oncol, 2012,30(17):2102-2111.

29 Fischer CA, Zlobec I, Green E, et al. Is the improved prognosis of p16positive oropharyngeal squamous cell carcinoma dependent of the treatment modality?. Int J Cancer, 2010,126(5): 1256-1262.

30 Rischin D, Young RJ, Fisher R, et al. Prognostic significance of p16INK4Aand human papillomavirus in patients with oropharyngeal cancer treated on TROG 02.02 phase III trial. J Clin Oncol, 2010,28(27):4142-4148.

31 Allen C T, Lewis JJ, El-Mofty SK, et al. Human papillomavirus and oropharynx cancer:biology, detection and clinical implications. Laryngoscope, 2010,120(9):1756-1772.

32 Spanos WC, Nowicki P, Lee DW, et al. Immune response during therapy with cisplatin or radiation for human papillomavirusrelated head and neck cancer. Arch Otolaryngol Head Neck Surg, 2009,135(11):1137-1146.

33 Vu HL, Sikora AG, Fu S, et al. HPV-induced oropharyngeal cancer,immune response and response to therapy. Cancer Lett, 2010,288(2):149-155.

34 Committee on Infectious Diseases. HPV vaccine recommendations. Pediatrics, 2012,129(3): 602-605. 